\title{
The Problem of Two Bodies with a Finite Velocity of Gravity
}

\author{
Vasyl Slyusarchuk \\ Department of Higher Mathematics \\ National University of Water and Enviromental Engineering \\ Rivne, Ukraine \\ e-mail: V.E.Slyusarchuk@gmail.com
}

\begin{abstract}
From the moment of Newton's discovery of the law of universal gravitation, ordinary differential equations were used to study the motion of bodies, since it was assumed that the velocity of gravitation is infinite. However, in reality the velocity of gravity is finite, which is consistent with the theory of relativity of Einstein, which postulated that the velocity of gravity matches the velocity of light, and the studies conducted by S. Kopeikin and E. Fomalont on the fundamental limit of the velocity of gravity. Due to the delay of the gravitational field for studying the motion of bodies, the mathematical apparatus based on differential equations with a delay argument is the most acceptable one. These equations are used to construct and study the mathematical model of the motion of two bodies. It is shown that the motion of these bodies with identical masses (with finite velocity of gravity!) is not carried out in accordance with Kepler's laws.
\end{abstract}

Keywords: two-body problem, two-body problem with finite gravity velocity.

\section{INTRODUCTION}

The article is devoted to the application of differential equations with a delayed argument to the construction and study of a mathematical model of the motion of two bodies under the action of the forces of the law of gravity, taking into account the finite speed of gravity.

The application to the study of the motion of two bodies of these equations is natural, since the gravitational effect of one body on another cannot be instantaneous and it takes time during which the gravitational field passes the distance between these bodies.

\section{TWO-BODY PROBLEM IN CLASSICAL CELESTIAL MECHANICS}

Since the discovery of the law of universal gravitation by I. Newton (1643-1727) in 1687, ordinary differential equations were used to study the motion of bodies, since it was believed that the speed of gravity is infinite.
The simplest task of classical celestial mechanics is the two-body problem. According to Newton's second law and the law of universal gravitation, the differential equations of motion of the bodies of this problem in some fixed Cartesian coordinate system have the form

$$
\left\{\begin{array}{l}
\ddot{\vec{r}}_{1}(t)=-\frac{G m_{2}}{\left|\vec{r}_{2}(t)-\vec{r}_{1}(t)\right|^{3}}\left(\vec{r}_{1}(t)-\vec{r}_{2}(t)\right), \\
\ddot{\vec{r}}_{2}(t)=-\frac{G m_{1}}{\left|\vec{r}_{2}(t)-\vec{r}_{1}(t)\right|^{3}}\left(\vec{r}_{2}(t)-\vec{r}_{1}(t)\right),
\end{array}\right.
$$

where $G$ is the gravitational constant, $m_{1}$ and $m_{1}$ are the masses of bodies, and $\left|\vec{r}_{2}(t)-\vec{r}_{1}(t)\right|$ is the Euclidean length of the vector $\vec{r}_{2}(t)-\vec{r}_{1}(t)$.

The study of system (1) is given in many works (see, for example, [1]-[3]).

I. Newton found a general solution to system (1) and this solution is given a geometric shape. The trajectories of the motion of one body relative to another and relative to the center of mass are finite sections.

\section{A MATHEMATiCAL MODEL OF THE MOTiON OF TWO BODIES, TAKING INTO ACCOUNT THE FINITE SPEED OF GRAVITY}

In real space, the speed of gravity cannot be infinite, as in Newton's theory. This statement is consistent with Einstein's theory of relativity, in which it is postulated that the speed of gravity coincides with the speed of light $c$, and with the studies of Kopeikin S.M. and Fomalont E.B. on the fundamental limit of the speed of gravity [4]. Application of this property of gravity allows you to build a model of motion of two bodies, which uses not ordinary differential equations, but differential equations with a delayed argument, and find new properties of the motion of these bodies.

Consider the movement of points $M_{1}$ and $M_{2}$ with masses $m_{1}$ and $m_{2}$ in a rectangular coordinate system $x, y, z$ with the origin at point $O$. We consider the coordinate system to be inertial. The position of points $M_{1}$ and $M_{2}$ at time $t$ is determined by their vectors $\vec{r}_{i}(t), i=\overline{1,2}$. 
If the speed of gravity were infinite, as in classical celestial mechanics, then, on the basis of the law of universal gravitation at time $t$, point $M_{2}$ pulled point $M_{1}$ with the power of

$$
\vec{F}(t)=\frac{G m_{1} m_{2}}{\left|\vec{r}_{2}(t)-\vec{r}_{1}(t)\right|^{3}}\left(\vec{r}_{2}(t)-\vec{r}_{1}(t)\right) .
$$

However, due to the finite speed of gravity, another force acts on point $M_{1}$

$$
\vec{F}_{1}(t)=\frac{G m_{1} m_{2}}{\left|\vec{r}_{2}\left(t-\tau_{2}(t)\right)-\vec{r}_{1}(t)\right|^{3}}\left(\vec{r}_{2}\left(t-\tau_{2}(t)\right)-\vec{r}_{1}(t)\right)
$$

The delay of gravity $\tau_{2}(t)$ in $(3)$ is determined by the equality

$$
c \tau_{2}(t)=\left|\vec{r}_{2}\left(t-\tau_{2}(t)\right)-\vec{r}_{1}(t)\right|,
$$

where $c$ is the speed of gravity.

Indeed, let points $M_{2}$ and $M_{1}$ move along the corresponding trajectories, parts of which are shown in Fig. 1, with speeds $\vec{v}_{2}(t)=\dot{\vec{r}}_{2}(t)$ and $\vec{v}_{1}(t)=\dot{\vec{r}}_{1}(t)$ and at time $t-\tau_{2}(t)$, where $\tau_{2}(t)$ satisfies $(4)$, are at points $C$ and $D$, respectively. Over a period of time $\left[t-\tau_{2}(t), t\right]$, point $M_{2}$ will move from point $C$ to point $A$, and point $M_{1}$ will move from point $D$ to point $B$. This period of time is enough for the gravitational field to propagate from speed $c$ from point $C$ to point $B$. Therefore, at time $t$, point $B$ is not affected by force (2), but by force (3).

Similarly, based on the final gravity velocity, a force acts on point $M_{2}$

$$
\vec{F}_{2}(t)=\frac{G m_{1} m_{2}}{\left|\vec{r}_{1}\left(t-\tau_{1}(t)\right)-\vec{r}_{2}(t)\right|^{3}}\left(\vec{r}_{1}\left(t-\tau_{1}(t)\right)-\vec{r}_{2}(t)\right) .
$$

The delay of gravity $\tau_{1}(t)$ to $(5)$ is determined by the equality

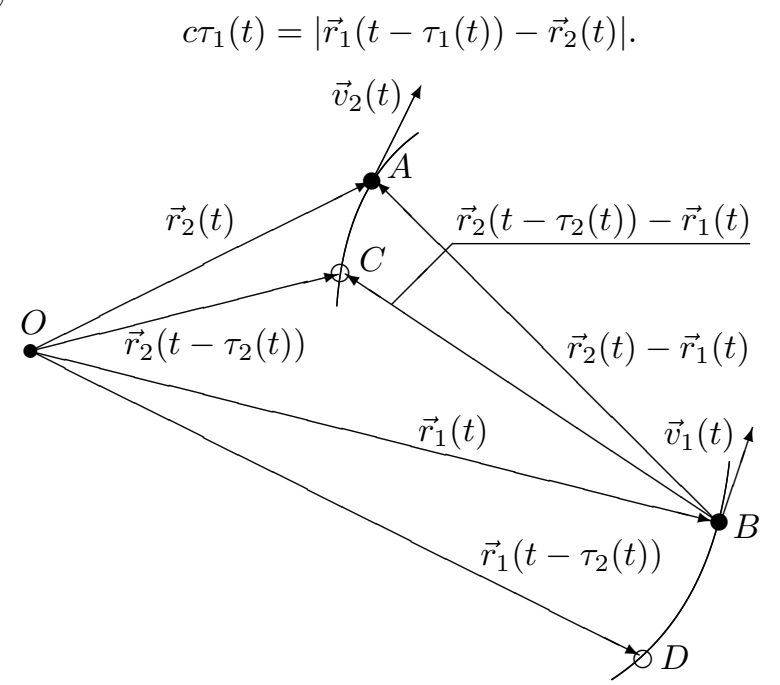

Fig. 1. Points $M_{1}$ and $M_{2}$ at time points $t-\tau_{2}(t)$ and $t$.

By virtue of implicit function theorems [5, pp. 449-453], functions $\tau_{2}(t)$ and $\tau_{1}(t)$ are continuous and differentiable.
From the above reflections, taking into account Newton's second law and the universal law, it follows that the motion of two points $M_{1}$ and $M_{2}$ with masses $m_{1}$ and $m_{2}$ is described by a system of equations

$$
\left\{\begin{array}{l}
m_{1} \ddot{\vec{r}}_{1}(t)=\vec{F}_{1}(t), \\
m_{2} \ddot{\vec{r}}_{2}(t)=\vec{F}_{2}(t) .
\end{array}\right.
$$

From here, (3) and (5) we get a system of equations that describes the motion of points $M_{1}$ and $M_{2}$. This system has the form

$$
\left\{\begin{array}{l}
\ddot{\bar{r}}_{1}(t)=\frac{G m_{2}}{\left|\vec{r}_{2}\left(t-\tau_{2}(t)\right)-\vec{r}_{1}(t)\right|^{3}}\left(\vec{r}_{2}\left(t-\tau_{2}(t)\right)-\vec{r}_{1}(t)\right), \\
\ddot{\vec{r}}_{2}(t)=\frac{G m_{1}}{\left|\vec{r}_{1}\left(t-\tau_{1}(t)\right)-\vec{r}_{2}(t)\right|^{3}}\left(\vec{r}_{1}\left(t-\tau_{1}(t)\right)-\vec{r}_{2}(t)\right) .
\end{array}\right.
$$

Note that system (7) is a system of differential equations with a retarded argument and differs significantly from system (1).

Elements of the theory of differential equations with delayed argument are presented in [6]-[9].

For a complete description of the motion of points $M_{1}$ and $M_{2}$, it is necessary for system (7) to use additional initial or boundary conditions (see problems 1 and 2).

Problem 1. We fix arbitrary time moment $t_{0}$ and continuous on segments $\left[t_{0}-\tau_{i}\left(t_{0}\right), t_{0}\right], i=\overline{1,2}$, vector functions $\vec{\varphi}_{0, i}(s)$ and $\vec{\varphi}_{1, i}(s), i=\overline{1,2}$, respectively. It is necessary to find solutions $\vec{r}_{i}(t), i=\overline{1,2}$, system $(7)$, which satisfy the initial conditions

$$
\begin{cases}\vec{r}_{i}(s)=\vec{\varphi}_{0, i}(s), & s \in\left[t_{0}-\tau_{i}\left(t_{0}\right), t_{0}\right], \\ \dot{\vec{r}}_{i}(s)=\vec{\varphi}_{1, i}(s), & s \in\left[t_{0}-\tau_{i}\left(t_{0}\right), t_{0}\right], \quad i=\overline{1,2} .\end{cases}
$$

Problem 2. Let $t_{1}$ and $t_{2}$ be arbitrary instants of time, for which $t_{1}<t_{2}-\tau_{i}\left(t_{2}\right), i=\overline{1,2}$. Consider twice continuously differentiated on segments $\left[t_{1}-\tau_{i}\left(t_{1}\right), t_{1}\right]$ and $\left[t_{2}-\tau_{i}\left(t_{2}\right), t_{2}\right], i=\overline{1,2}$, vector functions $\vec{\psi}_{1, i}(s)$ and $\vec{\psi}_{2, i}(s)$, $i=\overline{1,2}$, respectively. It is necessary to find solutions $\vec{r}_{i}(t)$, $i=\overline{1,2}$, system $(7)$ that satisfy the conditions

$$
\begin{cases}\vec{r}_{i}\left(s_{1}\right)=\vec{\psi}_{1, i}\left(s_{1}\right), & s_{1} \in\left[t_{1}-\tau_{i}\left(t_{1}\right), t_{1}\right], \\ \vec{r}_{i}\left(s_{2}\right)=\vec{\psi}_{2, i}\left(s_{2}\right), & s_{2} \in\left[t_{2}-\tau_{i}\left(t_{2}\right), t_{2}\right], \quad i=\overline{1,2}\end{cases}
$$

Equations (4), (6) and (7) with conditions (8) or (9) are mathematical models of the motion of two bodies, which take into account the speed of gravity.

\section{KERLER'S LAWS AND THE MAIN PURPOSE OF THE ARTICLE}

The movement of bodies (in particular, the movement of planets) in classical celestial mechanics is carried out according to the three laws of Kepler (1571-1630), discovered in 1605, 1601 and 1618, respectively [10, pp. 261, 262]: 


\section{Modeling, Control and Information Technologies - 2019}

1. The trajectories of the planets are ellipses, in one of the foci of which (common to all planets) is the Sun.

2. The areas that are described by the radius vectors of the planets drawn from the Sun increase in proportion to time.

3. The squares of the periods of rotation of the planets around the Sun are correlated as cubes of the major semiaxes of their orbits.

These laws in celestial mechanics (with a finite speed of gravity) have some inaccuracies.

Indeed, based on the delay of the gravitational field when the planets move around the Sun, the attracting point for each planet at a moment in time $t$ is not the center of the Sun, as in Kepler's first law, but another point in space in which the center of the Sun was at some moment in time $t-\tau(t)$, where $\tau(t)$ is a delay depending on the location of the planet and the Sun, similar to the delays $\tau_{2}(t)$ and $\tau_{1}(t)$, which satisfy (4) and (6).

Simple calculations show that each planet rotates around its "own" attracting point, which does not coincide with the center of the Sun, and these points do not coincide in pairs.

Therefore, due to the displacement of the attracting points, the motion of the bodies does not agree with Kepler's first law (with respect to attracting points).

It does not follow from this conclusion that ellipses cannot be trajectories of bodies.

The purpose of the article is to show that, based on the finite speed of gravity, the motion of bodies of the same mass cannot follow Kepler's laws.

\section{The Impossibility of MOVIng BOdies ALONG AN ELLIPSE BEHIND KEPLER'S SECOND LAW}

Consider the system of equations (7), which describes the motion of two points $M_{1}$ and $M_{2}$ with masses $m_{1}$ and $m_{2}$, taking into account the finite speed of gravity. Due to the presence of delays $\tau_{1}(t)$ and $\tau_{2}(t)$ in the equations, which is not in system (1), it is impossible to find a general solution to this system. However, with additional restrictions on the equations of the system, important properties of the motion of points can be obtained.

In the future, we assume that the masses of points $M_{1}$ and $M_{2}$ are the same and equal to $m$.

Suppose that points $M_{1}$ and $M_{2}$ move along the ellipse and beyond Kepler's second law.

Then the movement of point $M_{1}$ relative to point $M_{2}$ will be periodic.
We use the equations of motion of point $M_{1}$ relative to point $M_{2}$, i.e. equations for the vector function

$$
\vec{r}(t)=\vec{r}_{1}(t)-\vec{r}_{2}(t) .
$$

Since the points $M_{1}$ and $M_{2}$ are affected only by the forces arising from the interaction of these points, and these forces are equal in magnitude and opposite in direction, the accelerations $\ddot{\vec{r}}_{1}(t)$ and $\ddot{\vec{r}}_{2}(t)$ of the movement of the points have similar properties, i.e.

$$
\ddot{\vec{r}}_{1}(t)+\ddot{\vec{r}}_{2}(t) \equiv 0
$$

and therefore

$$
\dot{\vec{r}}_{1}(t)+\dot{\vec{r}}_{2}(t) \equiv \vec{v}
$$

where $\vec{v}$ is a constant vector. A ratio of (10) means that the center of the masses of points $M_{1}$ and $M_{2}$, which is determined by the vector function $\vec{r}_{O}(t)=\frac{1}{2}\left(\vec{r}_{1}(t)+\vec{r}_{2}(t)\right)$, moves in a straight line at a constant speed.

Without loss of generality, we can assume that the origin of the inertial coordinate system coincides with the center of mass of the points $M_{1}$ and $M_{2}$. Then the ratio of (10) is given in the form

$$
\dot{\vec{r}}_{1}(t)+\dot{\vec{r}}_{2}(t) \equiv 0
$$

Then $\vec{v}=0$ and, therefore, the speeds $\vec{v}_{1}(t)=\dot{\vec{r}}_{1}(t)$ and $\vec{v}_{2}(t)=\dot{\vec{r}}_{2}(t)$ of the movement of points $M_{1}$ and $M_{2}$ are opposite.

It follows from the above reasoning that points $M_{1}$ and $M_{2}$ are located on an ellipse with major axis $A_{1} A_{2}$ symmetrically with respect to its center $O$ (see Fig. 2).

The attracting points $M_{1}^{*}$ and $M_{2}^{*}$ for points $M_{2}$ and $M_{1}$, respectively, at time $t$ are also placed on the ellipse symmetrically with respect to its center $O$.

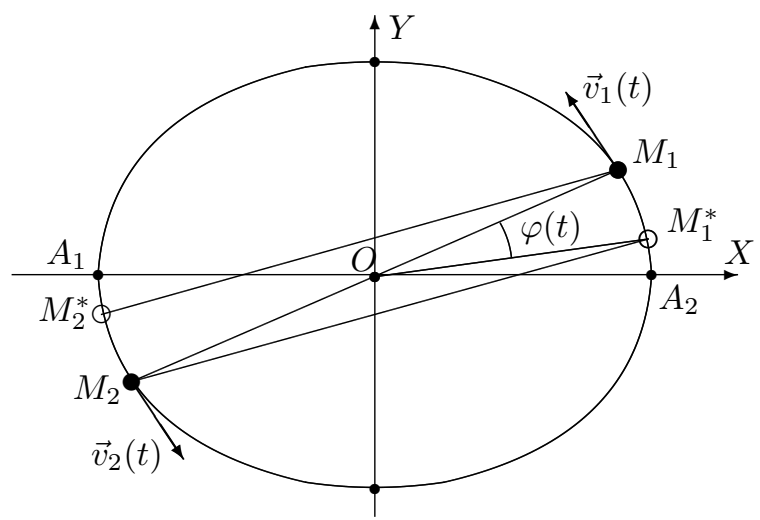

Fig. 2. Location of points $M_{1}, M_{2}, M_{1}^{*}$ and $M_{2}^{*}$.

The coordinates of points $M_{1}^{*}, M_{2}^{*}, M_{1}$ and $M_{2}$ at time $t$ are determined by vectors $\vec{r}_{1}\left(t-\tau_{1}(t)\right), \vec{r}_{2}\left(t-\tau_{2}(t)\right), \vec{r}_{1}(t)$ and $\vec{r}_{2}(t)$, respectively, and, obviously, according to Fig. 2 are carried out equalities

$$
\left|\overrightarrow{M_{1} M_{2}^{*}}\right|=\left|\overrightarrow{M_{2} M_{1}^{*}}\right|=
$$




$$
=\left|\vec{r}_{1}(t)-\vec{r}_{2}\left(t-\tau_{2}(t)\right)\right|=\left|\vec{r}_{2}(t)-\vec{r}_{1}\left(t-\tau_{1}(t)\right)\right| .
$$

Denote by $d(t)$ the length of the vector $\overrightarrow{M_{1} M_{2}^{*}}$.

Then, in system (7), delays $\tau_{1}(t)$ and $\tau_{2}(t)$ are the same (we denote them by $\tau(t)$ ), and according to the equations of this system, function $\vec{r}(t)$, which describes the motion of point $M_{1}$ relative to point $M_{2}$, is a solution to equation

$$
\ddot{\vec{r}}(t)=-\frac{G m}{d^{3}(t)}(\vec{r}(t)+\vec{r}(t-\tau(t))) .
$$

Next, we use a rectangular Cartesian coordinate system with the origin at the center of ellipse $O$. Let axis $O x$ of this coordinate system pass through the vertices $A_{1}$ and $A_{2}$ of the ellipse, and axis $O y$ be perpendicular to axis $O x$ and pass through point $O$.

Then function $\vec{r}(t)$ can be given in the form

$$
\vec{r}(t)=2(x(t), y(t)),
$$

where $x(t)$ and $y(t)$ are the coordinates of point $M_{1}$ at time $t$ and according to (11)

$$
\left\{\begin{array}{l}
\ddot{x}(t)=-\frac{G m}{d^{3}(t)}(x(t-\tau(t))+x(t)), \\
\ddot{y}(t)=-\frac{G m}{d^{3}(t)}(y(t-\tau(t))+y(t)) .
\end{array}\right.
$$

We pay attention to one important property of the solutions of the system of equations (13) using functions (12),

$$
\vec{v}(t)=\dot{\vec{r}}(t)=2(\dot{x}(t), \dot{y}(t))
$$

and

$$
v_{\sigma}(t)=2\left|\begin{array}{ll}
x(t) & y(t) \\
\dot{x}(t) & \dot{y}(t)
\end{array}\right| .
$$

In the case of classical celestial mechanics $v_{\sigma}(t)$ is a sectoral velocity of point $M_{1}$ relative to point $M_{2}$ and

$$
v_{\sigma}(t) \equiv v,
$$

where $v$ is a constant [11, p. 134].

Sectoral speed $v_{\sigma}(t)$ of the movement of point $M_{1}$ relative to point $M_{2}$, if we take into account the finiteness of the speed of gravity, is not a constant value, but strictly increases.

Indeed, given (13) and (14)

$$
\begin{gathered}
\frac{1}{2} \frac{d v_{\sigma}(t)}{d t}=\frac{d}{d t}\left|\begin{array}{ll}
x(t) & y(t) \\
\dot{x}(t) & \dot{y}(t)
\end{array}\right|=\left|\begin{array}{cc}
x(t) & y(t) \\
\ddot{x}(t) & \ddot{y}(t)
\end{array}\right|= \\
-\frac{G m}{d^{3}(t)}\left|\begin{array}{cc}
x(t) & y(t) \\
x(t-\tau(t))+x(t) & y(t-\tau(t))+y(t)
\end{array}\right|= \\
=-\frac{G m}{d^{3}(t)}\left|\begin{array}{cc}
x(t) & y(t) \\
x(t-\tau(t)) & y(t-\tau(t))
\end{array}\right| .
\end{gathered}
$$

From these equalities, in particular, we obtain the identity (15) when $\tau(t) \equiv 0$.

Let $t_{0}$ be an arbitrary moment of time from the interval during which the movement of points $M_{1}$ and $M_{2}$ occurs. Then for all $t \geqslant t_{0}$

$$
\frac{d}{d t}\left|\begin{array}{ll}
x(t) & y(t) \\
\dot{x}(t) & \dot{y}(t)
\end{array}\right|=\frac{G m}{d^{3}(t)} \Delta(t)
$$

where

$$
\Delta(t)=\left|\begin{array}{cc}
x(t-\tau(t)) & y(t-\tau(t)) \\
x(t) & y(t)
\end{array}\right| .
$$

We use Fig. 3, which shows the vectors $\vec{r}(t), \vec{v}(t), \vec{r}(t-$ $\tau(t))$ and part of the trajectory the motion of the point $M_{1}$ relative to the point $M_{2}$. The point $M_{3}$ in this figure is the end of the vector $\vec{v}(t)$.

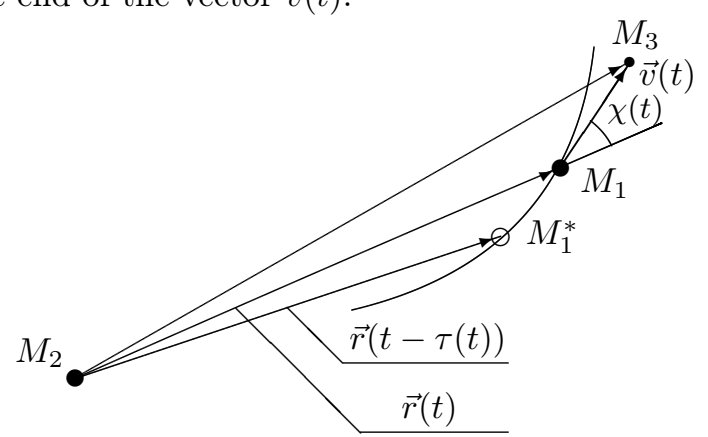

Fig. 3. To the growth of sectoral speed.

Hence the geometric meaning of the second-order determinant implies that the area $S_{M_{1} M_{2} M_{3}}(t)$ of triangle $M_{1} M_{2} M_{3}$ is a strictly increasing function and the rate of change of this area is proportional to the area $S_{M_{1} M_{2} M_{1}^{*}}(t)$ of triangle $M_{1} M_{2} M_{1}^{*}$, i.e.

$$
\frac{d S_{M_{1} M_{2} M_{3}}(t)}{d t}=\frac{G m}{d^{3}(t)} S_{M_{1} M_{2} M_{1}^{*}}(t), \quad t \geqslant t_{0} .
$$

The obtained property of the solutions of the system of equations (13) indicates an increase in the sectoral velocity of motion of point $M_{1}$ relative to point $M_{2}$ (the law of increasing the sectoral velocity).

An increase in this velocity contradicts the periodicity of the motion of point $M_{1}$ relative to point $M_{2}$.

Therefore, the assumption that points $M_{1}$ and $M_{2}$ move along the ellipse and under Kepler's second law is false.

Thus, according to Section IV, the movement of the point $M_{1}$ does not occur around the point $M_{2}$, but around the point of attraction $M_{2}^{*}$, and the first Kepler's law with respect to the point of attraction does not hold. Also, the sectoral velocity of movement of point $M_{1}$ relative to point $M_{2}$ is not constant but strictly increasing, and therefore the second Kepler law is not fulfilled.

To show that the third Kepler law is not fulfilled, it suffices to show that the trajectory of motion of point $M_{1}$ 


\section{Modeling, Control and Information Technologies - 2019}

relative to point $M_{2}$ is not an ellipse. Then, for points $M_{1}$ and $M_{2}$, the first Kepler law will not be fulfilled not only with respect to the attraction points, but also with respect to the motions of their motions.

\section{The Impossibility OF MOVING BOdIES ALONG AN ELLIPSE}

Suppose that points $M_{1}$ and $M_{2}$ move along the ellipse, as in Section V.

According on the increase in sector velocity $v_{\sigma}(t)$ and the movement of points $M_{1}$ and $M_{2}$ by ellipse for some numbers $\delta_{1}>0$ and $\delta_{2}>0$ for velocity $\vec{v}(t)$ of movement of point $M_{1}$ relative to point $M_{2}$ and for the angle $\chi(t)$ between vectors $\bar{r}(t)$ and $\vec{v}(t)$, the inequalities hold

$$
|\vec{v}(t)| \geqslant \delta_{1}, \quad t \geqslant t_{0}
$$

and

$$
\delta_{2} \leqslant \chi(t) \leqslant \pi-\delta_{2}, \quad t \geqslant t_{0} .
$$

Therefore, for some number $\delta_{3}>0$, the inequalities

$$
d\left(M_{1}, M_{1}^{*}\right) \geqslant \delta_{3}, \quad t \geqslant t_{0},
$$

will be satisfied for the distance $d\left(M_{1}, M_{1}^{*}\right)$ between points $M_{1}$ and $M_{1}^{*}$.

By virtue of (17) and the assumption that the points $M_{1}$ and $M_{1}^{*}$ move along the ellipse for a certain number $\delta_{4}>0$, the angle $\varphi(t)$ between vectors $\vec{r}_{1}(t)=\overrightarrow{O M_{1}}$ and $\vec{r}_{1}(t-\tau(t))=\overrightarrow{O M_{1}^{*}}$ (see Fig. 2) for each $t \geqslant t_{0}$ satisfies the inequality

$$
\frac{\pi}{2}>\varphi(t) \geqslant \delta_{4}
$$

Considering that the area $S(t)$ of the parallelogram constructed on $\vec{r}_{1}(t)$ and $\vec{r}_{1}(t-\tau(t))$ coincides with the value of the determinant $\Delta(t)$ and $S(t)=\left|\vec{r}_{1}(t)\right| \cdot \mid \vec{r}_{1}(t-$ $\tau(t)) \mid \sin \varphi(t)$ (see Fig. 2), we obtain that for some number $\delta_{5}>0$

$$
\Delta(t) \geqslant \delta_{5}, \quad t \geqslant t_{0} .
$$

Since $0<2 a \leqslant d(t) \leqslant 2 b, t \geqslant t_{0}$, where $a$ and $b$ are the lengths of the minor and major semi-axes of the ellipse (see Fig. 2), it is based on (16) and (18),

$$
\lim _{t \rightarrow+\infty} v_{\sigma}(t)=+\infty,
$$

which is impossible, since the speed cannot be greater than $c$.

Therefore, the assumption that points $M_{1}$ and $M_{2}$ move along an ellipse is false.

Thus, the motion of two bodies of the same mass in real space with a finite gravity velocity does not satisfy Kepler's laws.

\section{Additional REMARKS AND Literature}

The general case of a system of $n$ bodies, taking into account the finite speed of gravity, was considered by the author in article [12]. This article also pays attention to the construction of a mathematical model of the solar system with a finite speed of gravity and to obtain some new properties for it.

In [13], the problem of two bodies of arbitrary masses was studied taking into account the speed of gravity. The non-Kaplerian behavior and instability of the motion of these bodies are also shown.

In [14], systems of differential equations with delays and constraints on delays and derivatives of solutions, which are used in [12] and [13], are studied.

\section{REFERENCES}

[1] V. A. Brumberg, "Relativistic celestial mechanics", Moscow: Science, 1972.

[2] V. I. Arnold, V. V. Kozlov, A. N. Neishtadt, "Mathematical aspects of classical and celestial mechanics", Moscow: URSS, 2002.

[3] F. Moulyon, "An introduction to celestial mechanics", Moscow-Leningrad: ONTI NKTP, 1935.

[4] S. M. Kopeikin, E. Fomalont, "Fundamental limit of gravity velocity and its measurement", Earth and the Universe, 3, 2004.

[5] G. M. Fichtenholtz, "Course in Differential and Integral Calculus", Vol. 1, Moscow: Nauka, 1966.

[6] A. D. Myshkis, "Linear differential equations with retarded argument", Moscow-Leningrad: Gostekhizdat, 1951.

[7] R. Bellman, K. L. Cook, "Differential-difference equations", Moscow: Mir, 1967.

[8] L. E. Elsgolts, S. B. Norkin, "Introduction to the theory of differential equations with deviating argument", Moscow: Nauka, 1971.

[9] V. Yu. Slyusarchuk, "Absolute stability of dynamical systems with aftereffect", Rivne: NUWEE, 2003.

[10] Yu. A. Bely, "Johannes Kepler (1571-1630)", Moscow: Nauka, 1971.

[11] O. V. Golubeva, "Theothetic Mechanics", Moscow: Higher School, 1968.

[12] V. Yu. Slyusarchuk, "Mathematical model of the Solar system with account of gravitation velocity", Nonlinear Oscillations, 2018, vol. 21, № 2, pp. 238-261.

[13] V. Yu. Slyusarchuk, "Non-Keplerian behavior and instability of motion of two bodies caused by finite velocity of gravity", Nonlinear Oscillations, 2018, vol. 21, № 3, pp. 358-419.

[14] V. Yu. Slyusarchuk, "Investigation of systems of differential equations with delays and constraints imposed of the delays and derivatives of the solutions", Ukr. Mat. Zh., 2019, vol. 71, № 5, pp. 677-691. 